Acta Univ. Sapientiae, Mathematica, 6, 1 (2014) 61-72

DOI: $10.2478 /$ ausm-2014-0018

\title{
On some Ringel-Hall numbers in tame cases
}

\author{
Csaba Szántó \\ Babeş-Bolyai University, Cluj-Napoca \\ Faculty of Mathematics and Computer Science \\ email: szanto.cs@gmail.com
}

\begin{abstract}
Let $k$ be a finite field and consider the finite dimensional path algebra $k Q$, where $Q$ is a quiver of tame type i.e. of type $\tilde{A}_{n}, \tilde{D}_{n}, \tilde{E}_{6}, \tilde{E}_{7}, \tilde{E}_{8}$. Let $\mathcal{H}(\mathrm{kQ})$ be the corresponding Ringel-Hall algebra. We are going to determine the Ringel-Hall numbers of the form $\mathrm{F}_{\mathrm{XP}}^{\mathrm{P}^{\prime}}$ with $\mathrm{P}, \mathrm{P}^{\prime}$ preprojective

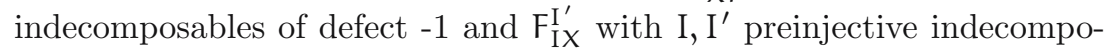
sables of defect 1 . It turns out that these numbers are either 1 or 0 .
\end{abstract}

\section{Introduction}

Let $k$ be a finite field with $q$ elements and consider the path algebra $k Q$ where $Q$ is a quiver of tame type i.e. of type $\tilde{\mathrm{A}}_{n}, \tilde{\mathrm{D}}_{n}, \tilde{\mathrm{E}}_{6}, \tilde{\mathrm{E}}_{7}, \tilde{\mathrm{E}}_{8}$. When $\mathrm{Q}$ is of type $\tilde{A}_{n}$ we exclude the cyclic orientation. So kQ is a finite dimensional tame hereditary algebra with the category of finite dimensional (hence finite) right modules denoted by mod-kQ. Let $[M]$ be the isomorphism class of $M \in$ mod-kQ. The category mod-kQ can and will be identified with the category rep- $\mathrm{QQ}$ of the finite dimensional $\mathrm{k}$-representations of the quiver $\mathrm{Q}=\left(\mathrm{Q}_{0}=\right.$ $\left.\{1,2, \ldots, n\}, Q_{1}\right)$. Here $Q_{0}=\{1,2, \ldots, n\}$ denotes the set of vertices of the quiver, $Q_{1}$ the set of arrows and for an arrow $\alpha$ we denote by $s(\alpha)$ the starting point of the arrow and by $e(\alpha)$ its endpoint. Recall that a k-representation of $\mathrm{Q}$ is defined as a set of finite dimensional $k$-spaces $\left\{V_{i} \mid i=\overline{1, n}\right\}$ corresponding to the vertices together with $k$-linear maps $V_{\alpha}: V_{s(\alpha)} \rightarrow V_{e(\alpha)}$ corresponding to the arrows. The dimension of a module $M=\left(V_{i}, V_{\alpha}\right) \in \bmod -k Q=$ rep- $k Q$

2010 Mathematics Subject Classification: 16G20

Key words and phrases: tame hereditary algebra, Ringel-Hall algebra, Ringel-Hall numbers 
is then $\underline{\operatorname{dim}} M=\left(\operatorname{dim}_{k} V_{i}\right)_{i=1, n} \in \mathbb{Z}^{n}$. For $a=\left(a_{i}\right), b=\left(b_{i}\right) \in \mathbb{Z}^{n}$ we say that $a \leq b$ iff $b_{i}-a_{i} \geq 0 \forall i$.

Let $\mathrm{P}(\mathfrak{i})$ and $\mathrm{I}(\boldsymbol{i})$ be the projective and injective indecomposable corresponding to the vertex $i$ and consider the Cartan matrix $C_{Q}$ with the $j$-th column being $\underline{\operatorname{dim} P}(\mathfrak{j})$. We have a biliniar form on $\mathbb{Z}^{n}$ defined as $\langle a, b\rangle=a C_{Q}^{-t} b^{t}$. Then for two modules $X, Y \in \bmod -k Q$ we get

$$
\langle\underline{\operatorname{dim} X}, \underline{\operatorname{dim}} Y\rangle=\operatorname{dim}_{k} \operatorname{Hom}(X, Y)-\operatorname{dim}_{k} \operatorname{Ext}^{1}(X, Y) .
$$

We denote by $\mathbf{q}$ the quadratic form defined by $\mathbf{q}(a)=\langle a, a\rangle$. Then $\mathbf{q}$ is positive semi-definite with radical $\mathbb{Z} \delta$, that is $\left\{a \in \mathbb{Z}^{n} \mid \mathbf{q}(a)=0\right\}=\mathbb{Z} \delta$. Here $\delta$ is known for each type $\tilde{\mathrm{A}}_{n}, \tilde{\mathrm{D}}_{n}, \tilde{\mathrm{E}}_{6}, \tilde{\mathrm{E}}_{7}, \tilde{\mathrm{E}}_{8}$ (see [4]). A vector $a \in \mathbb{N}^{n}$ is called positive real root of $\mathbf{q}$ if $\mathbf{q}(\mathbf{a})=1$. It is known (see [4]) that for all positive roots $a$ there is a unique indecomposable module $M \in \bmod -k Q$ (unique up to isomorphism) with $\underline{\operatorname{dim}} M=a$. The rest of the indecomposables are of dimension $t \delta$, with $t$ positive integers. The defect of a module $M$ is $\partial M=\langle\delta, \underline{\operatorname{dim} M}\rangle=-\langle\underline{\operatorname{dim}} M, \delta\rangle$. For a short exact sequence $0 \rightarrow X \rightarrow Y \rightarrow Z \rightarrow 0$ we have that $\partial Y=\partial X+\partial Z$.

Consider the Auslander-Reiten translates $\tau=\operatorname{DExt}^{1}(-, \mathrm{kQ})$ and $\tau^{-1}=$ $\operatorname{Ext}^{1}(D(k Q),-)$, where $D=\operatorname{Hom}_{k}(-, k)$. An indecomposable module $M$ is preprojective (preinjective) if exists a positive integer $m$ such that $\tau^{\mathrm{m}}(M)=0$ $\left(\tau^{-m}(M)=0\right)$. Otherwise $M$ is said to be regular. Note that the dimension vectors of preprojective and preinjective indecomposables are positive real roots of q. A module is preprojective (preinjective, regular) if every indecomposable component is preprojective (preinjective, regular). Note that an indecomposable module $M$ is preprojective (preinjective, regular) iff $\partial M<0(\partial M>0$, $\partial M=0)$. Moreover if $Q$ is of type $\tilde{A}_{n}$ then $\partial M=-1$ for $M$ preprojective indecomposable and $\partial M=1$ for $M$ preinjective indecomposable.

We consider now the rational Ringel-Hall algebra $\mathcal{H}(\mathrm{kQ})$ of the algebra $k Q$. Its $\mathbb{Q}$-basis is formed by the isomorphism classes $[M]$ from mod-kQ and the multiplication is defined by

$$
\left[\mathrm{N}_{1}\right]\left[\mathrm{N}_{2}\right]=\sum_{[\mathrm{M}]} \mathrm{F}_{\mathrm{N}_{1} \mathrm{~N}_{2}}^{\mathrm{M}}[\mathrm{M}]
$$

The structure constants $\mathrm{F}_{\mathrm{N}_{1} \mathrm{~N}_{2}}^{\mathrm{M}}=\left|\left\{\mathrm{M} \supseteq \mathrm{U} \mid \mathrm{U} \cong \mathrm{N}_{2}, \mathrm{M} / \mathrm{U} \cong \mathrm{N}_{1}\right\}\right|$ are called Ringel-Hall numbers. It is well-known that Ringel-Hall algebras play an important role in linking representation theory with the theory of quantum groups. They also appear in cluster theory. This is why it is important to know the structure of these algebras, by deriving formulas for Ringel-Hall numbers.

When $\mathrm{Q}$ is the Kronecker quiver (i.e. of type $\tilde{A}_{1}$ ) then the Ringel-Hall numbers were determined in [7] and [3]. It was shown that for $\mathrm{P}, \mathrm{P}^{\prime}$ preprojective 
indecomposables the Ringel-Hall numbers $F_{X P}^{P^{\prime}}$ are 0 or 1 . A dual statement could be formulated for preinjectives. This result was important since it played a crucial role in obtaining other formulas for Ringel-Hall numbers.

Our main theorem generalizes this result for every tame case. More precisely we show that the Ringel-Hall numbers of the form $\mathrm{F}_{X \mathrm{P}}^{\mathrm{P}^{\prime}}$ with $\mathrm{P}, \mathrm{P}^{\prime}$ preprojective indecomposables of defect -1 and $\mathrm{F}_{\mathrm{IX}}^{\mathrm{I}^{\prime}}$ with $\mathrm{I}, \mathrm{I}^{\prime}$ preinjective indecomposables of defect 1 are either 1 or 0 . We also describe the modules $X$ for which these Ringel-Hall numbers are 1.

We should remark that the main result of this paper is a fundamental tool in obtaining other important formulas for the Ringel-Hall products in tame cases (see also the paper [9]).

Finally we note that the left to right implication part of Lemma 4 appears as main result in [10], however for the sake of completeness we include the full proof of it.

\section{Facts on tame hereditary algebras}

For a detailed description of the forthcoming notions we refer to [1],[2],[4],[6] and [12].

Let $k$ be a finite field with $q$ elements and consider the path algebra $k Q$ where $\mathrm{Q}$ is a quiver of tame type.

The vertices of the Auslander-Reiten quiver of kQ are the isomorphism classes of indecomposables and its arrows correspond to the so-called irreducible maps. It will have a preprojective component (with all the isoclasses of preprojective indecomposables), a preinjective component (with all the isoclasses of preinjective indecomposables). All the other components (containing the isoclasses of regular indecomposables) are "tubes" of the form $\mathbb{Z} A_{\infty} / \mathrm{m}$, where $m$ is the rank of the tube. The tubes are indexed by the points of the scheme $\mathbb{P}_{k}^{1}$, the degree of a point $x \in \mathbb{P}_{k}^{1}$ being denoted by deg $x$. A tube of rank 1 is called homogeneous, otherwise it is called non-homogeneous. We have at most 3 non-homogeneous tubes indexed by points $x$ of degree $\operatorname{deg} x=1$. All the other tubes are homogeneous. Notice that the number of points $x \in \mathbb{P}_{k}^{1}$ of degree 1 is $q+1$ and there are $N(q, l)=\frac{1}{l} \sum_{d \mid l} \mu\left(\frac{l}{d}\right) q^{d}$ points of degree $l \geq 2$, where $\mu$ is the Möbius function and $N(q, l)$ is the number of monic, irreducible polynomials of degree $l$ over a field with q elements (see [12]).

Indecomposables from different tubes have no nonzero homomorphisms and no non-trivial extensions. Note that all regular modules form an extensionclosed abelian subcategory of mod-kQ, the simple objects in this subcate- 
gory being called quasi-simple modules; any indecomposable regular module is regular uniserial and hence it is uniquely determined by its quasi-socle and quasi-length, and also by its quasi-top and quasi-length.

In case of a homogeneous tube $\tau_{x}$ we have a single quasi-simple regular denoted by $R_{x}[1]$ with $\underline{\operatorname{dim}} R_{x}[1]=(\operatorname{deg} x) \delta$, which lies on the "mouth" of the tube. $R_{x}[t]$ will denote the regular indecomposable with quasi-socle $R_{x}[1]$ and quasi-length $t$. In case of a non-homogeneous tube $\tau_{x}$ of rank $m$ on the mouth of the tube we have $m$ quasi-simples denoted by $R_{x}^{i}[1] i=\overline{1, m}$ such that $\sum_{i=1}^{m} \underline{\operatorname{dim}} R_{x}^{i}[1]=\delta . R_{x}^{i}[t]$ will denote the regular indecomposable with quasi-socle $R_{x}^{i}[1]$ and quasi-length $t$.

The following lemma is well-known.

Lemma 1 a) For $\mathrm{P}$ preprojective, I preinjective, and $\mathrm{R}$ regular module we have $\operatorname{Hom}(R, P)=\operatorname{Hom}(I, P)=\operatorname{Hom}(I, R)=\operatorname{Ext}^{1}(P, R)=\operatorname{Ext}^{1}(P, I)=\operatorname{Ext}^{1}(R, I)=$ 0 .

b) If $\mathrm{x} \neq \mathrm{x}^{\prime}$ and $\mathrm{R}_{\mathrm{x}}\left(\mathrm{R}_{\mathrm{x}^{\prime}}\right)$ is a regular with components from the tube $\tau_{\mathrm{x}}$ $\left(\tau_{x^{\prime}}\right)$, then $\operatorname{Hom}\left(R_{x}, R_{x^{\prime}}\right)=\operatorname{Ext}^{1}\left(R_{x}, R_{x^{\prime}}\right)=0$.

c) For $\tau_{x}$ homogeneous and $R_{x}[t], R_{x}\left[t^{\prime}\right]$ indecomposables from $\tau_{x}$ we have $\operatorname{dim}_{k} \operatorname{Hom}\left(R_{x}[t], R_{x}\left[t^{\prime}\right]\right)=\operatorname{dim}_{k} \operatorname{Ext}^{1}\left(R_{x}[t], R_{x}\left[t^{\prime}\right]\right)=\min \left(t, t^{\prime}\right) \operatorname{deg} x$.

d) For $\tau_{x}$ non-homogeneous of rank $\mathrm{m}$ and $\mathrm{R}_{\mathrm{x}}^{\mathrm{i}}[\mathrm{t}]$ an indecomposable from $\tau_{\mathrm{x}}$ such that $\mathrm{lm}<\mathrm{t} \leq(\mathrm{l}+1) \mathrm{m}$ we have $\operatorname{dim}_{\mathrm{k}} \operatorname{End}\left(\mathrm{R}_{\mathrm{x}}^{\mathrm{i}}[\mathrm{t}]\right)=\mathrm{l}+1$.

e) For $\tau_{x}$ non-homogeneous of rank $\mathrm{m}$ and $\mathrm{R}_{x}^{\mathrm{i}}[\mathrm{t}]$ an indecomposable from $\tau_{x}$ such that $\mathrm{lm} \leq \mathrm{t}<(\mathrm{l}+1) \mathrm{m}$ we have $\operatorname{dim}_{\mathrm{k}} \operatorname{Ext}^{1}\left(\mathrm{R}_{x}^{\mathrm{i}}[\mathrm{t}], \mathrm{R}_{x}^{\mathrm{i}}[\mathrm{t}]\right)=\mathrm{l}$.

f) For $\mathrm{P}$ preprojective and I preinjective indecomposable modules we have $\operatorname{End}(\mathrm{P}) \cong k, \operatorname{End}(\mathrm{I}) \cong k,|\operatorname{Aut}(\mathrm{P})|=|\operatorname{Aut}(\mathrm{I})|=\mathrm{q}-1$ and $\operatorname{Ext}^{1}(\mathrm{P}, \mathrm{P})=$ $\operatorname{Ext}^{1}(\mathrm{I}, \mathrm{I})=0$.

\section{Some Ringel-Hall numbers}

Consider the Ringel-Hall numbers of the form $\mathrm{F}_{\mathrm{XP}}^{\mathrm{P}^{\prime}}$ with $\mathrm{P}, \mathrm{P}^{\prime}$ preprojetive indecomposables of defect -1 and $\mathrm{F}_{\mathrm{IX}}^{\mathrm{I}^{\prime}}$ with $\mathrm{I}, \mathrm{I}^{\prime}$ preinjective indecomposables of defect 1 . We are going to show that these numbers are either 1 or 0 .

We consider the preprojective case, the preinjective case being dual. We begin with some lemmas. The first lemma is well known (see for example in [11]).

Lemma 2 Let $\mathrm{P}$ be a preprojective indecomposable with defect $\partial \mathrm{P}=-1, \mathrm{P}^{\prime}$ a preprojective module and $\mathrm{R}$ a regular indecomposable. Then we have:

a) Every nonzero morphism $\mathrm{f}: \mathrm{P} \rightarrow \mathrm{P}^{\prime}$ is a monomorphism. 
b) For every nonzero morphism $\mathrm{f}: \mathrm{P} \rightarrow \mathrm{R}, \mathrm{f}$ is either a monomorphism or $\operatorname{Im} \mathrm{f}$ is regular. In particular if $\mathrm{R}$ is quasi-simple and $\operatorname{Im} \mathrm{f}$ is regular then $\mathrm{f}$ is an epimorphism.

Proof. a) Consider the short exact sequence $0 \rightarrow \operatorname{Ker} f \rightarrow P \rightarrow \operatorname{Im} f \rightarrow 0$. Since $\operatorname{Ker} f \subseteq P$ and $\operatorname{Im} f \subseteq P^{\prime}$ we have that Ker $f$ and $\operatorname{Im} f$ are either preprojective (so with negative defect) or 0 . Moreover we have that $\partial \operatorname{Ker} f+\partial \operatorname{Im} f=\partial P=$ -1 and we know that $\operatorname{Im} f \neq 0$ (since $f$ is nonzero). It follows that $\operatorname{Ker} f=0$.

b) Consider the short exact sequence $0 \rightarrow \operatorname{Ker} f \rightarrow P \rightarrow \operatorname{Im} f \rightarrow 0$. Since $\operatorname{Ker} f \subseteq P$ we have that $\operatorname{Ker} f$ is either preprojective (so with negative defect) or 0 . On the other hand $\operatorname{Im} f \subseteq R$ implies that $\operatorname{Im} f$ can contain preprojectives and regulars as direct summands (and it is nonzero since $f$ is nonzero). The equality $\partial \operatorname{Ker} f+\partial \operatorname{Im} f=\partial P=-1$ gives us two cases. When $\partial \operatorname{Ker} f=0$ then Ker $f$ is 0 so $f$ is monomorphism. In the second case (when $\partial \operatorname{Ker} f=-1$ ) $\partial \operatorname{Im} f=0$, so $\operatorname{Im} f$ can contain just regular direct summands.

Lemma 3 Let $\mathrm{P}$ be a preprojective indecomposable with defect $\partial \mathrm{P}=-1$ (Then $\underline{\operatorname{dim} P} \neq \delta$ since $\underline{\operatorname{dim} P}$ is a positive real root of $\mathbf{q})$.

a) Suppose that $\operatorname{dim} \mathrm{P}>\delta$. Then $\mathrm{P}$ projects to the quasi-simple regular $\mathrm{R}_{\mathrm{x}}[1]$ from each homogeneous tube $\tau_{x}$ with $(\operatorname{deg} x) \delta<\operatorname{dim} \mathrm{P}$. Also $\mathrm{P}$ projects to a unique quasi-simple regular from the mouth of each non-homogeneous tube $\tau_{\mathrm{x}}$. We will denote these quasi-simple regulars by $\mathrm{R}_{x}^{\mathrm{P}}[1]$ where for $\tau_{x}$ homogeneous with $(\operatorname{deg} x) \delta<\operatorname{dim} P$ we have $\mathrm{R}_{x}^{\mathrm{P}}[1]=\mathrm{R}_{\mathrm{x}}[1]$.

b) Suppose that $\operatorname{dim} \mathrm{P}<\delta$. Then $\mathrm{P}$ projects at most to a single quasi-simple regular from each non-homogeneous tube $\tau_{x}$ denoted by $\mathrm{R}_{x}^{\mathrm{P}}[1]$.

Proof. a) Suppose that $R_{x}[1]$ denotes the quasi-simple regular from the mouth of the homogeneous tube $\tau_{x}$ with $\underline{\operatorname{dim}} R_{x}[1]=(\operatorname{deg} x) \delta<\underline{\operatorname{dim} P}$. Then we have $\operatorname{Ext}^{1}\left(P, R_{x}[1]\right)=0$ (see Lemma 1$)$ so

$$
\begin{gathered}
\operatorname{dim}_{k} \operatorname{Hom}\left(P, R_{\chi}[1]\right)=\left\langle\underline{\operatorname{dim} P}, \underline{\operatorname{dim} R_{\chi}}[1]\right\rangle=\langle\underline{\operatorname{dim} P},(\operatorname{deg} x) \delta\rangle= \\
(\operatorname{deg} x)(-\partial P)=\operatorname{deg} x \neq 0 .
\end{gathered}
$$

This means that we have a nonzero morphism $f: P \rightarrow R_{x}[1]$ with $\underline{\operatorname{dim} P}>$ $\underline{\operatorname{dim}} R_{\chi}[1]$. Using Lemma 2 we deduce that $f$ is not a monomorphism, so $\operatorname{Im} f$ is regular and $R_{\chi}[1]$ is quasi-simple, which means that $f$ is an epimorphism.

Denote by $R_{x}^{i}[1], i=\overline{1, m}$ the $i$-th quasi-simple regular from the mouth of the non-homogeneous tube $\tau_{x}$ of rank $m \geq 2$. Notice that this time $\operatorname{deg} x=1$, 
$\sum_{i=1}^{m} \underline{\operatorname{dim}} R_{x}^{i}[1]=\delta$ and $\operatorname{Ext}^{1}\left(P, R_{x}^{i}[1]\right)=0$, so we have

$$
\begin{aligned}
& \sum_{i=1}^{m} \operatorname{dim}_{k} \operatorname{Hom}\left(P, R_{x}^{i}[1]\right)=\sum_{i=1}^{m}\left\langle\underline{\operatorname{dim} P}, \underline{\operatorname{dim}} R_{x}^{i}[1]\right\rangle \\
& =\left\langle\underline{\operatorname{dim} P}, \sum_{i=1}^{m} \underline{\operatorname{dim}} R_{\chi}^{i}[1]\right\rangle=\langle\underline{\operatorname{dim} P}, \delta\rangle=-\partial P=1 .
\end{aligned}
$$

It follows that $\exists$ ! $i_{0}$ such that $\operatorname{Hom}\left(P, R_{x}^{i_{0}}[1]\right) \neq 0$, so we have a nonzero morphism $f: P \rightarrow R_{x}^{i_{0}}[1]$ with $\underline{\operatorname{dim} P}>\delta>\underline{\operatorname{dim}} R_{x}^{i_{0}}[1]$. Using Lemma 2 we deduce that $f$ is not a monomorphism, so $\operatorname{Im} f$ is regular and $R_{x}^{\mathfrak{i}_{0}}[1]$ is quasi-simple, which means that $f$ is an epimorphism. Let $R_{x}^{P}[1]:=R_{x}^{\mathfrak{i}_{0}}[1]$.

b) Since $\underline{\operatorname{dim} P}<\delta$ clearly $\mathrm{P}$ could project only on quasi-simple regulars from non-homogeneous tubes. Denote again by $R_{x}^{i}[1], i=\overline{1, m}$ the $i$-th quasi-simple regular on the mouth of the non-homogeneous tube $\tau_{x}$ of rank $m \geq 2$. As above we can deduce that $\exists ! i_{0}$ such that $\operatorname{Hom}\left(P, R_{x}^{i_{0}}[1]\right) \neq 0$, so we have a nonzero morphism $f: P \rightarrow R_{x}^{\mathfrak{i}_{0}}[1]$. But if $\underline{\operatorname{dim} P} \ngtr \underline{\operatorname{dim}} R_{x}^{i_{0}}[1]$ then $f$ is a monomorphism and not an epimorphism.

Remark 1 Notice that $\operatorname{dim}_{\mathrm{k}} \operatorname{Hom}\left(\mathrm{P}, \mathrm{R}_{\chi}^{\mathrm{P}}[1]\right)=\operatorname{deg} \chi$.

Lemma 4 Let $\mathrm{P} ¥ \mathrm{P}^{\prime}$ be preprojective indecomposables with defect -1 . Then $\mathrm{F}_{\mathrm{XP}}^{\mathrm{P}^{\prime}} \neq 0$ iff $\mathrm{X}$ satisfies the following conditions:

i) it is a regular module with $\underline{\operatorname{dim} X}=\underline{\operatorname{dim}} \mathrm{P}^{\prime}-\underline{\operatorname{dim} P}$;

ii) if it has an indecomposable component from a tube $\tau_{x}$ then the quasi-top of this component is the quasi-simple regular $\mathrm{R}_{x}^{\mathrm{P}^{\prime}}[1]$;

iii) its indecomposable components are taken from pairwise different tubes.

Proof. " $\Rightarrow$ " Suppose $\mathrm{F}_{X P}^{P^{\prime}} \neq 0$. We will check the conditions i), ii) and iii).

Condition i). Since $\mathrm{F}_{X P}^{\mathrm{P}^{\prime}} \neq 0$ we have a short exact sequence $0 \rightarrow \mathrm{P} \rightarrow \mathrm{P}^{\prime} \rightarrow$ $X \rightarrow 0$. Then $\underline{\operatorname{dim}} X=\underline{\operatorname{dim}} P^{\prime}-\underline{\operatorname{dim}} P$ and $\partial P^{\prime}=\partial P+\partial X$, but $\partial P^{\prime}=\partial P=-1$, so $\partial X=0$. Notice that $X$ can't have preprojective components, for if $P^{\prime \prime}$ would be such a component then $\mathrm{P}^{\prime} \rightarrow \mathrm{P}^{\prime \prime} ¥ \mathrm{P}^{\prime}$ which is impossible due to Lemma 2 a). So $X$ is regular.

Condition ii). Let $R$ be an indecomposable component of $X$ taken from the tube $\tau_{x}$. Denote by topR its quasi-top which must be quasi-simple due to uniseriality. Then $\mathrm{P}^{\prime} \rightarrow \mathrm{X} \rightarrow \mathrm{R} \rightarrow$ top $\mathrm{R}$ so using Lemma 3 top $\mathrm{R} \cong \mathrm{R}_{x}^{\mathrm{P}^{\prime}}[1]$.

Condition iii). Suppose $X=X^{\prime} \oplus R_{1} \oplus \ldots \oplus R_{l}$, where $R_{1}, \ldots, R_{l}$ are taken from the same tube $\tau_{x}$. Then by Condition ii) they have the same quasi-top 
$R_{x}^{P^{\prime}}[1]$ and we have the monomorphism

$$
0 \rightarrow \operatorname{Hom}\left(X, R_{x}^{P^{\prime}}[1]\right) \rightarrow \operatorname{Hom}\left(P^{\prime}, R_{x}^{P^{\prime}}[1]\right) .
$$

It follows that

$$
\operatorname{dim}_{k} \operatorname{Hom}\left(X, R_{x}^{P^{\prime}}[1]\right) \leq \operatorname{dim}_{k} \operatorname{Hom}\left(P^{\prime}, R_{x}^{P^{\prime}}[1]\right)=\operatorname{deg} x .
$$

We can conclude that

$\operatorname{dim}_{k} \operatorname{Hom}\left(X, R_{x}^{P^{\prime}}[1]\right)=\operatorname{dim}_{k} \operatorname{Hom}\left(X^{\prime}, R_{x}^{P^{\prime}}[1]\right)+\sum_{i=1}^{l} \operatorname{dim}_{k} \operatorname{Hom}\left(R_{i}, R_{x}^{P^{\prime}}[1]\right) \leq \operatorname{deg} \chi$, $\operatorname{dim}_{k} \operatorname{Hom}\left(R_{i}, R_{x}^{P^{\prime}}[1]\right)=\operatorname{deg} x$ for $\tau_{x}$ homogeneous

and

$$
\operatorname{dim}_{k} \operatorname{Hom}\left(R_{i}, R_{x}^{P^{\prime}}[1]\right) \geq 1=\operatorname{deg} x \text { for } \tau_{x} \text { non-homogeneous. }
$$

It follows that $l=1$.

" $\Leftarrow$ " Let $\mathrm{R}$ be an indecomposable regular module with $\underline{\operatorname{dim} R}<\underline{\operatorname{dim}} \mathrm{P}^{\prime}$ satisfying condition ii). By Lemma $2 \mathrm{~b}$ ) it follows that for a nonzero morphism $f: P^{\prime} \rightarrow R, \operatorname{Im} f$ is regular. We will show that $P^{\prime}$ projects on $R$. Observe that if $R=R_{x}^{P^{\prime}}[1]$ the assertion is true due to Lemma 3. Suppose now that $R$ is not a quasi-simple.

If $R$ is from a homogeneous tube $\tau_{x}$ then $R=R_{x}[t], \underline{\operatorname{dim} R}=t(\operatorname{deg} x) \delta$ and $\operatorname{Hom}\left(P^{\prime}, R\right) \neq 0$ since $\operatorname{dim}_{k} \operatorname{Hom}\left(P^{\prime}, R\right)=\left\langle\underline{\operatorname{dim}} P^{\prime}, t(\operatorname{deg} x) \delta\right\rangle=-t(\operatorname{deg} x) \partial P^{\prime}=$ $t \operatorname{deg} x$. Notice that in the case when there are no epimorphisms in $\operatorname{Hom}\left(P^{\prime}, R\right)$ then using Lemma $2 \mathrm{~b}$ ) and the uniseriality of regulars we would have $\operatorname{Hom}\left(P^{\prime}, R\right)=\operatorname{Hom}\left(P^{\prime}, R_{x}[t]\right) \cong \operatorname{Hom}\left(P^{\prime}, R_{x}[t-1]\right)$, a contradiction. So we have an epimorphism $P^{\prime} \rightarrow R$.

If $R$ is from a non-homogeneous tube $\tau_{x}$ of rank $m$ then $\operatorname{deg} x=1, R=R_{x}^{j}[t]$ and top $R=R_{x}^{P^{\prime}}[1]=R_{x}^{i}[1]\left(\right.$ condition ii)). We have that $\underline{\operatorname{dim} R}=\underline{\operatorname{dim}} R_{x}^{j}[t-1]+$ $\underline{\operatorname{dim}}(\operatorname{top} R)$, so $\operatorname{dim}_{k} \operatorname{Hom}\left(P^{\prime}, R\right)=\left\langle\underline{\operatorname{dim}} P^{\prime}, \underline{\operatorname{dim}} R_{x}^{j}[t-1]\right\rangle+\left\langle\underline{\operatorname{dim}} P^{\prime}, \underline{\operatorname{dim}}(\operatorname{top} R)\right\rangle=$ $\operatorname{dim}_{k} \operatorname{Hom}\left(P^{\prime}, R_{x}^{j}[t-1]\right)+1>0$. If there is no epimorphism $P^{\prime} \rightarrow R$ then using uniseriality and Lemma $2 b$ ) for nonzero $f \in \operatorname{Hom}\left(P^{\prime}, R\right)$ we have that $\operatorname{Im} f=$ $R_{x}^{j}[l]$ with $1 \leq l<t$ and $P^{\prime}$ projects on top $\operatorname{Im} f$ so top $R_{x}^{j}[l]=\operatorname{top} R_{x}^{j}[t]=\operatorname{top} R$ (see Lemma 3). But this means that $t-l=s m$ with $s \geq 1$ so if $t \leq m$ we have a contradiction and if $t>m$ as in the homogeneous case we would have that $\operatorname{Hom}\left(P^{\prime}, R_{x}^{j}[t]\right) \cong \operatorname{Hom}\left(P^{\prime}, R_{x}^{j}[t-m]\right)$ that is

$$
0=\left\langle\underline{\operatorname{dim}} P^{\prime}, \underline{\operatorname{dim}} R_{x}^{j}[t]-\underline{\operatorname{dim}} R_{x}^{j}[t-m]\right\rangle=\left\langle\underline{\operatorname{dim}} P^{\prime}, \delta\right\rangle=1,
$$

again a contradiction. 
Suppose now that the module $X=R_{1} \oplus \ldots \oplus R_{l}$ satisfies conditions i), ii) and iii). From the discussion above we have the epimorphisms $f_{i}: P^{\prime} \rightarrow R_{i}$. Let $f: P^{\prime} \rightarrow X, f(x)=\sum f_{i}(x)$ the diagonal map. Due to Lemma 2 b) we have that $\operatorname{Im} f$ is regular, so due to uniseriality $\operatorname{Im} f=R_{1}^{\prime} \oplus \ldots \oplus R_{l}^{\prime}$ with $R_{i}^{\prime} \subseteq R_{i}$. Since $f_{i}=p_{i} f$ are epimorphisms we have that $R_{i}^{\prime}=R_{i}$, so $f$ is an epimorphism. Notice that Ker $f \subseteq P^{\prime}$ hence it is preprojective, $\partial \operatorname{Ker} f=\partial P^{\prime}-\partial X=-1$ therefore Ker $f$ is an indecomposable preprojective with $\underline{\operatorname{dim} K e r f=\underline{\operatorname{dim} P}}$. It follows that $\operatorname{Ker} \mathrm{f} \cong \mathrm{P}$, so we have an exact sequence $0 \rightarrow \mathrm{P} \rightarrow \mathrm{P}^{\prime} \rightarrow \mathrm{X} \rightarrow 0$ which implies that $F_{X P}^{P^{\prime}} \neq 0$.

Lemma 5 Let $\mathrm{P} ¥ \mathrm{P}^{\prime}$ be preprojective indecomposables with defect -1 such that $\operatorname{Hom}\left(\mathrm{P}, \mathrm{P}^{\prime}\right) \neq 0$. Suppose the points $\mathrm{y}_{\mathrm{i}} \in \mathbb{P}_{\mathrm{k}}^{1}, i=\overline{1, s}(\mathrm{~s}=0,1,2,3)$ are indexing the non-homogeneous tubes (in case $\mathrm{s}=0$ we have only homogeneous tubes). Then $\underline{\operatorname{dim}} \mathrm{P}^{\prime}-\underline{\operatorname{dim}} \mathrm{P}=\mathrm{t}_{0} \delta+\sum_{i=1}^{s} \sigma_{i}^{0}$, where $0 \leq \sigma_{i}^{0}<\delta$ and $\sigma_{i}^{0}$ (in case it is nonzero) is the dimension of a regular from the non-homogeneous tube $\tau_{y_{i}}$ with top $\mathrm{R}_{y_{i}}^{P^{\prime}}[1]$. In this case $\operatorname{dim}_{k} \operatorname{Hom}\left(\mathrm{P}, \mathrm{P}^{\prime}\right)=\mathrm{t}_{0}+1$ so $\mathrm{t}_{0}$ is unique.

Proof. Since $\operatorname{Hom}\left(P, \mathrm{P}^{\prime}\right) \neq 0$ we have a monomorphism $\mathrm{P} \rightarrow \mathrm{P}^{\prime}$ with factor $\mathrm{X}$ satisfying conditions i),ii),iii) from the previous lemma. It follows that dim $P^{\prime}-$ $\underline{\operatorname{dim}} P=\underline{\operatorname{dim}} X=t \delta+\sum_{i=1}^{s} \sigma_{i}$, where $0 \leq \sigma_{i}$ (in case it is nonzero) is the dimension of a regular $R_{i}$ from the non-homogeneous tube $\tau_{y_{i}}$ with top $R_{y_{i}}^{P^{\prime}}[1]$. Suppose $\sigma_{i}=t_{i} \delta+\sigma_{i}^{0}$ with $0 \leq \sigma_{i}^{0}<\delta$ and $0 \leq t_{i}$. If $t_{i} \neq 0$ then there is a unique regular $R_{t_{i}}$ of dimension $t_{i} \delta$ from the non-homogeneous tube $\tau_{y_{i}}$ which embeds into $R_{i}$; the factor will be of dimension $\sigma_{i}^{0}$ with top $R_{y_{i}}^{P^{\prime}}[1]\left(\right.$ if $\left.\sigma_{i}^{0} \neq 0\right)$. Let $t_{0}=t+\sum_{i=1}^{s} t_{i}$.

We show that $\operatorname{dim}_{k} \operatorname{Hom}\left(P, P^{\prime}\right)=t_{0}+1$. Suppose first that we don't have non-homogeneous tubes, so we are in the Kronecker case (see [12]). In this case $\delta=(1,1), \underline{\operatorname{dim}} P^{\prime}-\underline{\operatorname{dim}} P=t_{0} \delta$ and then $\operatorname{dim}_{k} \operatorname{Hom}\left(P, P^{\prime}\right)=t_{0}+1$. (see for example [7] Lemma 2.1). Consider now the case when we do have nonhomogeneous tubes, so $s \geq 1$ and suppose $t_{0} \delta+\sigma_{1}^{0} \neq 0$. Then there are unique regular indecomposables $R_{1} \in \tau_{y_{1}}$ of dimension $t_{0} \delta+\sigma_{1}^{0}$ and top $R_{y_{1}}^{P^{\prime}}[1]$ and $R_{i} \in \tau_{y_{i}}$ of dimension $\sigma_{i}^{0}$ and top $R_{y_{i}}^{P^{\prime}}[1]$ for $i \in I=\left\{i=\overline{2, s} \mid \sigma_{i}^{0} \neq 0\right\}$. Suppose that $I^{\prime}=\left\{i=\overline{1, s} \mid \sigma_{i}^{0} \neq 0\right\}$ and $\left|I^{\prime}\right|=l$ (where we can have $l=0$ ). Let $R=R_{1} \oplus\left(\bigoplus_{i \in I} R_{i}\right)$. It follows from the previous lemma that $F_{R P}^{P^{\prime}} \neq 0$ so we have a short exact sequence $0 \rightarrow \mathrm{P} \rightarrow \mathrm{P}^{\prime} \rightarrow \mathrm{R} \rightarrow 0$ which induces the exact sequences

$$
0 \rightarrow \operatorname{End}(\mathrm{P}) \rightarrow \operatorname{Hom}\left(\mathrm{P}, \mathrm{P}^{\prime}\right) \rightarrow \operatorname{Hom}(\mathrm{P}, \mathrm{R}) \rightarrow \operatorname{Ext}^{1}(\mathrm{P}, \mathrm{P})
$$


and

$$
0 \rightarrow \operatorname{End}(R) \rightarrow \operatorname{Hom}\left(P^{\prime}, R\right) \rightarrow \operatorname{Hom}(P, R) \rightarrow \operatorname{Ext}^{1}(R, R) \rightarrow \operatorname{Ext}^{1}\left(P^{\prime}, R\right) .
$$

We deduce using Lemma 1 and Remark 1 that

$$
\begin{gathered}
\operatorname{dim}_{k} \operatorname{Hom}\left(P, P^{\prime}\right)=\operatorname{dim}_{k} \operatorname{Hom}(P, R)+1= \\
\operatorname{dim}_{k} \operatorname{Hom}\left(P^{\prime}, R\right)+\operatorname{dim}_{k} \operatorname{Ext}^{1}(R, R)-\operatorname{dim}_{k} \operatorname{End}(R)+1,
\end{gathered}
$$

where

$$
\begin{gathered}
\operatorname{dim}_{k} \operatorname{Hom}\left(P^{\prime}, R\right)=\left\langle\underline{\operatorname{dim}} P^{\prime}, \underline{\operatorname{dim} R}\right\rangle=\left\langle\underline{\operatorname{dim}} P^{\prime}, t_{0} \delta+\sum_{i=1}^{s} \sigma_{i}^{0}\right\rangle=t_{0}+l, \\
\operatorname{dim}_{k} \operatorname{Ext}^{1}(R, R)=\operatorname{dim}_{k} \operatorname{Ext}^{1}\left(R_{1}, R_{1}\right)+\sum_{i \in I} \operatorname{dim}_{k} \operatorname{Ext}^{1}\left(R_{i}, R_{i}\right)=t_{0}, \\
\operatorname{dim}_{k} \operatorname{End}(R)=\operatorname{dim}_{k} \operatorname{End}\left(R_{1}\right)+\sum_{i \in I} \operatorname{dim}_{k} \operatorname{End}\left(R_{i}\right)=t_{0}+l,
\end{gathered}
$$

so it results that $\operatorname{dim}_{k} \operatorname{Hom}\left(P, P^{\prime}\right)=t_{0}+1$.

The following lemma can be found in [5] or in [8].

Lemma 6 For to nonnegative integer we have that

$$
\begin{aligned}
& \sum_{\substack{\left(t_{x}\right)_{x \in \mathbb{P}_{k}^{1}} \\
t_{x} \in \mathbb{Z}, t_{x} \geq 0 \\
\sum_{x} t_{x}(\operatorname{deg} x)=t_{0}}} 1=\frac{q^{t_{0}+1}-1}{q-1} .
\end{aligned}
$$

Now we are ready to prove the main theorem.

Theorem 1 Let $\mathrm{P} ¥ \mathrm{P}^{\prime}$ be preprojective indecomposables with defect -1 . If $\operatorname{Hom}\left(\mathrm{P}, \mathrm{P}^{\prime}\right)=0$ then $\mathrm{F}_{\mathrm{XP}}^{\mathrm{P}^{\prime}}=0$ for every $\mathrm{X}$. If $\operatorname{Hom}\left(\mathrm{P}, \mathrm{P}^{\prime}\right) \neq 0$ then $\mathrm{F}_{\mathrm{XP}}^{\mathrm{P}^{\prime}}=1$ for any $\mathrm{X}$ satisfying conditions $i)$, ii) and iii) from Lemma 4 , otherwise $\mathrm{F}_{\mathrm{XP}}^{\mathrm{P}^{\prime}}=0$.

Proof. Suppose $\operatorname{Hom}\left(\mathrm{P}, \mathrm{P}^{\prime}\right) \neq 0$. Then using the notation from Lemma 5 $\underline{\operatorname{dim}} P^{\prime}-\underline{\operatorname{dim}} P=t_{0} \delta+\sum_{i=1}^{s} \sigma_{i}^{0}$, where $0 \leq \sigma_{i}^{0}<\delta$ and $\sigma_{i}^{0}$ (in case it is nonzero) is the dimension of a regular from the non-homogeneous tube $\tau_{y_{i}}$ with top $R_{y_{i}}^{P^{\prime}}[1]$; also we have $\operatorname{dim}_{k} \operatorname{Hom}\left(P, P^{\prime}\right)=t_{0}+1$. Since by Lemma 2 every nonzero 
morphism in $\operatorname{Hom}\left(P, \mathrm{P}^{\prime}\right)$ is a monomorphism and $|\operatorname{Aut}(\mathrm{P})|=\mathrm{q}-1$. We have that the number of submodules of $\mathrm{P}^{\prime}$ which are isomorphic to $\mathrm{P}$ is

$$
u_{P}^{P^{\prime}}=\frac{\left|\operatorname{Hom}\left(P, P^{\prime}\right)\right|-1}{|\operatorname{Aut}(P)|}=\frac{q^{t_{0}+1}-1}{q-1} .
$$

A regular module $X$ satisfying conditions i), ii) and iii) from Lemma 4 will be called of good type. By Lemma 4 we have that

$$
\mathrm{u}_{\mathrm{P}}^{\mathrm{P}^{\prime}}=\sum_{[\mathrm{X}]} \mathrm{F}_{\mathrm{XP}}^{\mathrm{P}^{\prime}}=\sum_{\mathrm{X} \text { of good type }}^{\sum_{\mathrm{X}]}} \mathrm{F}_{\mathrm{XP}}^{\mathrm{P}^{\prime},}
$$

the terms in the last sum being nonzero. We will count now the number of nonisomorphic regulars of good type. For $\tau_{x}$ a homogeneous tube and $t \geq 1$ denote by $R_{x}(t)$ the regular $R_{x}[t]$ of quasi-length $t$ and let $R_{x}(0)=0$. For $\tau_{y_{i}}$ $(i=\overline{1, s})$ a non-homogeneous tube and $t \neq 0$ denote by $R_{y_{i}}(t)$ the unique indecomposable from $\tau_{y_{i}}$ of dimension $t \delta+\sigma_{i}^{0}$ with top $R_{y_{i}}^{P^{\prime}}[1]$. For $t=0$ and $\sigma_{i}^{0} \neq 0$ let $R_{y_{i}}(0)$ be the unique indecomposable from $\tau_{y_{i}}$ of dimension $\sigma_{i}^{0}$ with top $R_{y_{i}}^{P^{\prime}}[1]$. For $t=0$ and $\sigma_{i}^{0}=0$ let $R_{y_{i}}(0)=0$. Then the modules

$$
\begin{gathered}
\bigoplus \mathrm{R}_{x}\left(\mathrm{t}_{x}\right) \\
\left.\mathrm{t}_{x}\right)_{x \in \mathbb{P}_{k}^{1}} \\
\mathrm{t}_{x} \in \mathbb{Z}, \mathrm{t}_{x} \geq 0 \\
\sum_{x} \mathrm{t}_{x}(\operatorname{deg} x)=\mathrm{t}_{0}
\end{gathered}
$$

are nonisomorphic regulars of good type, so by the previous lemma we have at least $\frac{\mathrm{q}^{\mathrm{t}^{+}+1}-1}{\mathrm{q}-1}$ of them. It follows that

$$
\frac{\mathrm{q}^{\mathrm{t}_{0}+1}-1}{\mathrm{q}-1}=\sum_{X \text { of good type }}^{\sum_{\mathrm{X}]}} \mathrm{F}_{\mathrm{XP}}^{\mathrm{P}^{\prime}}
$$

the number of nonzero terms in the sum being at least $\frac{\mathrm{q}^{\mathrm{t}^{+}+1}-1}{\mathrm{q}-1}$, so the assertion of the theorem follows.

Remark 2 It follows from the previous theorem that for $\mathrm{P} ¥ \mathrm{P}^{\prime}$ preprojective indecomposables with defect -1 such that $\operatorname{Hom}\left(\mathrm{P}, \mathrm{P}^{\prime}\right) \neq 0$ the decomposition from Lemma $5 \underline{\operatorname{dim}} \mathrm{P}^{\prime}-\underline{\operatorname{dim} P}=\mathrm{t}_{0} \delta+\sum_{i=1}^{s} \sigma_{i}^{0}$ (where $0 \leq \sigma_{i}^{0}<\delta$ and $\sigma_{i}^{0}$ (in case it is nonzero) is the dimension of a regular from the non-homogeneous tube $\tau_{y_{i}}$ with top $\left.\mathrm{R}_{y_{i}}^{\mathrm{P}^{\prime}}[1]\right)$ is unique, so both $\mathrm{t}_{0}$ and $\sigma_{i}^{0}$ are unique. 
We can dualize the previous results for preinjective modules. So we have the dual of Lemma 3.

Lemma 7 Let $\mathrm{I}$ be a preinjective indecomposable with defect $\partial \mathrm{I}=1$.

a) Suppose that $\underline{\operatorname{dimI}}>\delta$. Then the quasi-simple regular $\mathrm{R}_{\mathrm{x}}[1]$ from each homogeneous tube $\tau_{\mathrm{x}}$ with $(\operatorname{deg} \mathrm{x}) \delta<\operatorname{dim} \mathrm{I}$ embeds into I. Also, a unique quasisimple regular from the mouth of each non-homogeneous tube $\tau_{x}$ embeds into I. We will denote these quasi-simple regulars by $\mathrm{R}_{x}^{\mathrm{I}}[1]$, where for $\tau_{x}$ homogeneous with $(\operatorname{deg} x) \delta<\operatorname{dim} I$ we have $\mathrm{R}_{x}^{\mathrm{I}}[1]=\mathrm{R}_{\chi}[1]$.

b) Suppose that $\underline{\operatorname{dim} I}<\delta$. Then at most a single quasi-simple regular from each non-homogeneous tube $\tau_{x}$ embeds into I. We denote this quasi-simple regular by $\mathrm{R}_{x}^{\mathrm{I}}[1]$.

The dual of Theorem 1 is

Theorem 2 Let $\mathrm{I} ¥ \mathrm{I}^{\prime}$ be preinjective indecomposables with defect 1 .

If $\operatorname{Hom}\left(\mathrm{I}^{\prime}, \mathrm{I}\right)=0$ then $\mathrm{F}_{\mathrm{IX}}^{\mathrm{I}^{\prime}}=0$ for every $\mathrm{X}$. If $\operatorname{Hom}\left(\mathrm{I}^{\prime}, \mathrm{I}\right) \neq 0$ then $\mathrm{F}_{\mathrm{IX}}^{\mathrm{I}^{\prime}}=1$ for $\mathrm{X}$ satisfying the conditions $i$ ), ii) and iii) below, otherwise $\mathrm{F}_{\mathrm{IX}}^{\mathrm{I}^{\prime}}=0$.

i) $\mathrm{X}$ is a regular module with $\underline{\operatorname{dim} X}=\underline{\operatorname{dim}} \mathrm{P}^{\prime}-\underline{\operatorname{dim} P}$;

ii) If $\mathrm{X}$ has an indecomposable component from a tube $\tau_{\mathrm{x}}$ then the quasi-socle of this component is the quasi-simple regular $\mathrm{R}_{x}^{\mathrm{I}^{\prime}}[1]$;

iii) The indecomposable components of $\mathrm{X}$ are taken from pairwise different tubes.

\section{Acknowledgements}

This work was supported by the Bolyai Scholarship of the Hungarian Academy of Sciences.

\section{References}

[1] I. Assem, D. Simson, A. Skowronski, Elements of representation theory of associative algebras, Volume 1: Techniques of representation theory, LMS Student Texts (No. 65), Cambridge Univ. Press 2006.

[2] M. Auslander, I. Reiten, S. Smalø, Representation theory of Artin algebras, Cambridge Stud. in Adv. Math. 36, Cambridge Univ. Press 1995.

[3] P. Baumann, C. Kassel, The Hall algebra of the category of coherent sheaves on the projective line, J. Reine Angew. Math., 533 (2001), 207233. 
[4] V. Dlab, C. M. Ringel, Indecomposable representations of graphs and algebras, Mem. Am. Math. Soc., 173 (1976).

[5] A. Hubery, The composition algebra of an affine quiver, preprint, http://arxiv.org/abs/math/0403206

[6] C. M. Ringel, Tame algebras and integral quadratic forms, Lect. Notes Math. 1099, Springer 1984.

[7] Cs. Szántó, Hall numbers and the composition algebra of the Kronecker algebra, Algebr. Represent. Theory, 9 (2006), 465-495.

[8] Cs. Szántó, Hall coefficients in the Kronecker case, Proceedings of the Algebra Symposium, "Babes-Bolyai" University Cluj-Napoca, November 23-24 (2001), 253-260.

[9] Cs. Szántó, On some Ringel-Hall products in tame cases, J. Pure Appl. Algebra, 216 (2012), 2069-2078.

[10] Cs. Szántó, On some nonzero Ringel-Hall numbers in tame cases, Mathematica (Cluj), 53 (76) (2011), 189-195.

[11] P. Zhang, Composition algebras of affine type, J. Algebra, 206 (1998), 505-540.

[12] P. Zhang, Y. B. Zhang, J. Y. Guo, Minimal generators of RingelHall algebras of affine quivers, J. Algebra, 239 (2001), 675-704. 